\title{
High Energy Beam Lifetime Analysis
}

\author{
R.H. Howell \\ P.A. Sterne \\ J. Hartley \\ T.E. Cowan
}

This paper was prepared for submittal to the

\section{1th International Conference on Positron Annihilation \\ Kansas City, MO \\ May 24-30, 1997}

May 1997

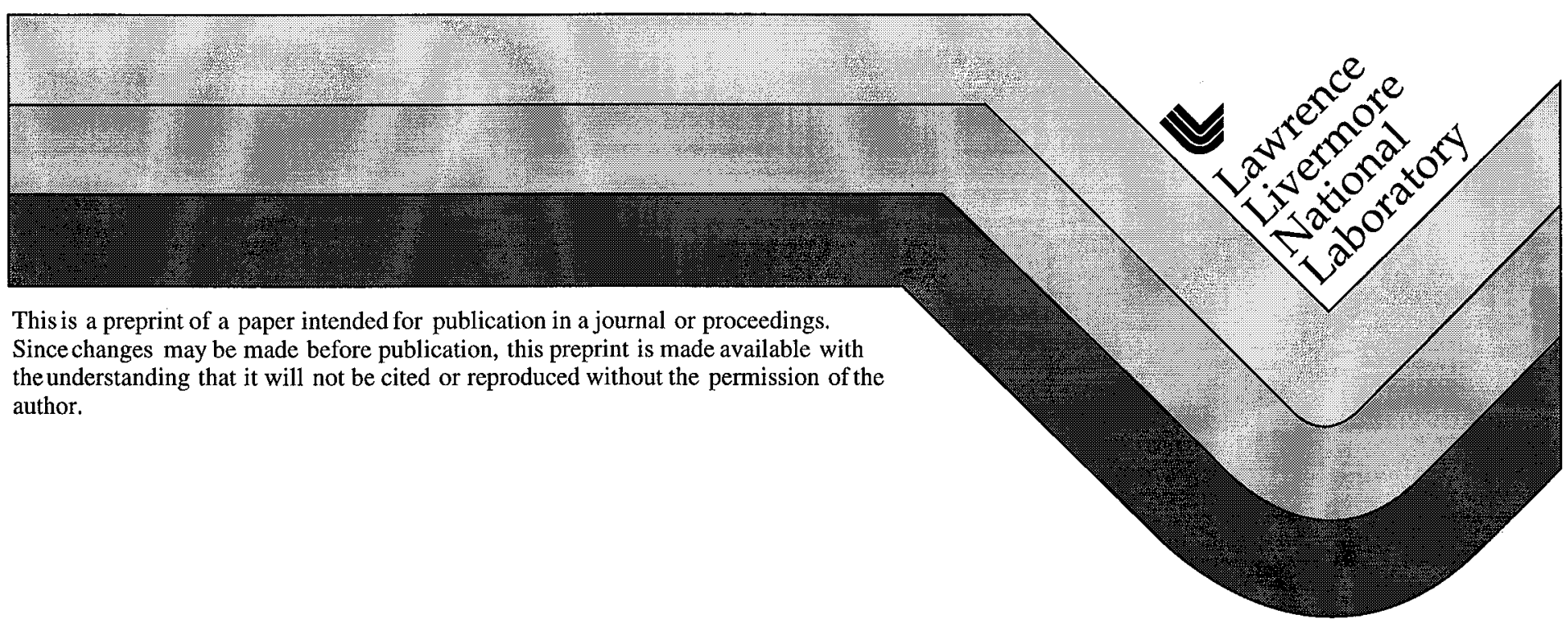




\section{DISCLAIMER}

This document was prepared as an account of work sponsored by an agency of the United States Government. Neither the United States Government nor the University of California nor any of their employees, makes any warranty, express or implied, or assumes any legal liability or responsibility for the accuracy, completeness, or usefulness of any information, apparatus, product, or process disclosed, or represents that its use would not infringe privately owned rights. Reference herein to any specific commercial product, process, or service by trade name, trademark, manufacturer, or otherwise, does not necessarily constitute or imply its endorsement, recommendation, or favoring by the United States Government or the University of California. The views and opinions of authors expressed herein do not necessarily state or reflect those of the United States Government or the University of California, and shall not be used for advertising or product endorsement purposes. 


\title{
High Energy Beam Lifetime Analysis
}

\author{
R. H. Howell, P. A. Sterne, J. Hartley, and T. E. Cowan \\ Lawrence Livermore National Laboratory, Livermore CA 94550 USA
}

We have developed a positron lifetime defect analysis capability based on a $3 \mathrm{MeV}$ electrostatic accelerator. The high energy beam lifetime spectrometer is operational with a $60 \mathrm{mCi}{ }^{22} \mathrm{Na}$ source providing a current of $710^{5}$ positrons per second. Lifetime data are derived from a thin plastic transmission detector providing an implantation time and $\mathrm{a} \mathrm{BaF}_{2}$ detector to determine the annihilation time. Positron lifetime analysis is performed with a $3 \mathrm{MeV}$ positron beam on thick sample specimens at counting rates in excess of 2000 per second. The instrument is being used for bulk sample analysis and analysis of samples encapsulated in controlled environments for in situ measurements.

\section{Introduction}

Positron annihilation lifetime spectroscopy has been limited by long measurement times and restrictive requirements on the sample geometry. Significant advantages can be achieved by performing positron measurements with a monoenergetic beam. With a beam only single sample pieces are required and there is a relaxation of the sample geometry so that engineering samples can be measured. Better definition of the measurement volume can be obtained by controlling the energy and direction of the positrons during implantation in the sample. For high energies the positron beam can even pass through thin windows before implanting into the sample allowing in situ measurements with samples at temperature or under stress. In positron annihilation lifetime spectroscopy higher data rates are achieved by a positron beam due to the nearly $100 \%$ efficiency in determining the implantation time. Also in a well controlled geometry only annihilation events from the sample will contribute to the data which eliminates systematic contributions from the positron source and simplifies the data analysis.

\section{High Energy Beam}

There are both low and high energy positron beams available at LLNL. New developments in experiments using our intense, low energy beam are found in an accompanying article in this conference. Our high energy beam is derived from a 60 $\mathrm{mCi}{ }^{22} \mathrm{Na}$ source moderated by a 1 micron thick tungsten single crystal foil positioned in the terminal of a $3 \mathrm{MeV}$ Pelletron electrostatic accelerator, see fig. 1 . The source and terminal focusing geometry is close coupled to the accelerator column. Both moderated positrons and positrons directly emitted from the source are captured and accelerated. This beam contains a current of $510^{5} \mathrm{e}^{+} \mathrm{s}^{-1}$ captured directly from the radioactive source at $3 \mathrm{MeV}$ and a similar current, $3.510^{5} \mathrm{e}^{+} \mathrm{s}^{-1}$, of positrons emitted in a narrow phase space 
from the moderator foil. Both the beam from moderated positrons and the beam from direct capture can be focused by a magnetic lens to a $3 \mathrm{~mm}$ spot at a location $2 \mathrm{~m}$ from the exit of the Pelletron. However only the beam from the moderator foil is well conditioned and only it can be transported to other experimental stations to perform other experiments such as high energy positron channeling.

Positron lifetime spectroscopy is performed at the first focus at the end of the Pelletron and uses both moderated and direct positrons. Lifetimes are measured by determining the time of implantation for each positron as it passes through a $2 \mathrm{~mm}$ plastic scintillator and the time of annihilation from annihilation gamma-rays detected by a $\mathrm{BaF}_{2}$ detector. This results in a highly efficient lifetime spectrometer with reduced accidental backgrounds and a system resolution of less than 250 ps. Positrons leave the implantation detector with a broad energy distribution, an average energy of $2.6 \mathrm{MeV}$ and a $1 \mathrm{~cm}$ effective beam diameter at a sample placed $4 \mathrm{~cm}$ beyond the $2 \mathrm{~mm}$ plastic detector. At this energy positrons implant from $\mathrm{mm}$ to $\mathrm{cm}$ into a sample depending on the sample density. Positrons that scatter away from the sample are rejected by an anticoincidence detector. In the pictured close coupled geometry a small, $<1 \%$, contribution from annihilations in the detector system is found in measured spectra and 2000 counts per second can be achieved. The annihilation detector can also be run in coincidence with a second detector to constrain all events to annihilation in the sample. This geometry reduces the counting rates to less than 1000 counts per second but eliminates all systematic contributions with lifetimes greater than $0.25 \mathrm{~ns}$. This system is modeled on the one used successfully at Stuttgart Germany[1]

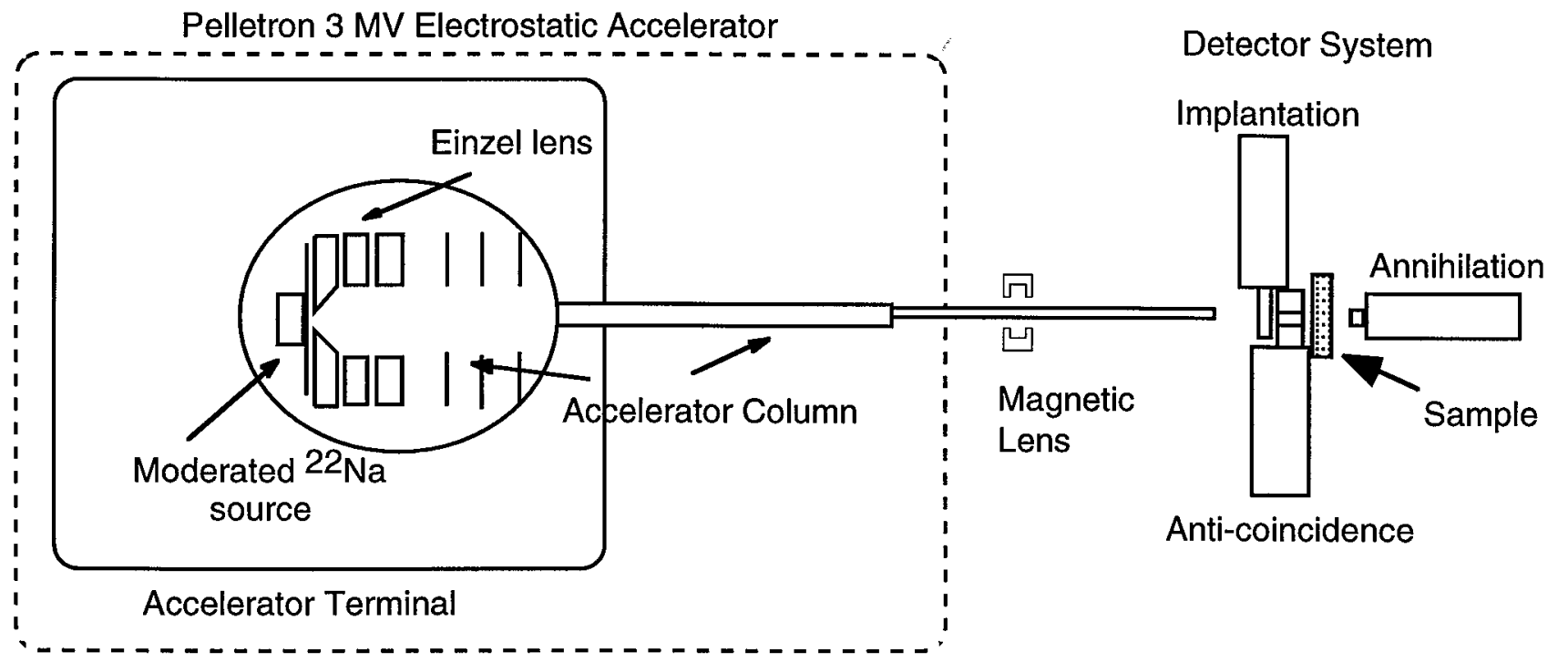

Figure 1. Schematic diagram of the critical components of the high energy beam positron annihilation lifetime system.

We are using positron annihilation lifetime spectroscopy with the high energy beam to determine aging effects in carbon fiber resin composites. We are measuring the changes 
in the hole volume brought on by accelerated aging of the composites at elevated temperature and in hostile atmospheres. Data describing changes in the hole volume are correlated with infrared spectroscopy and mechanical tests to provide a complete description of the changes during aging. We are also using the high energy beam to determine changes in polymeric and molecular solids brought on by irradiation or chemical degradation. The simple sample geometry allows us to measure samples directly obtained from mechanical testing experiments. Also the absence of background contributions in the lifetime range above $0.25 \mathrm{~ns}$ allows us to look at composite materials containing small resin fractions. Also with our increased sensitivity we can see ns positron lifetimes in resins and their composites previously thought to be free of positronium, such as polyimides ( Kapton, and K3B ).

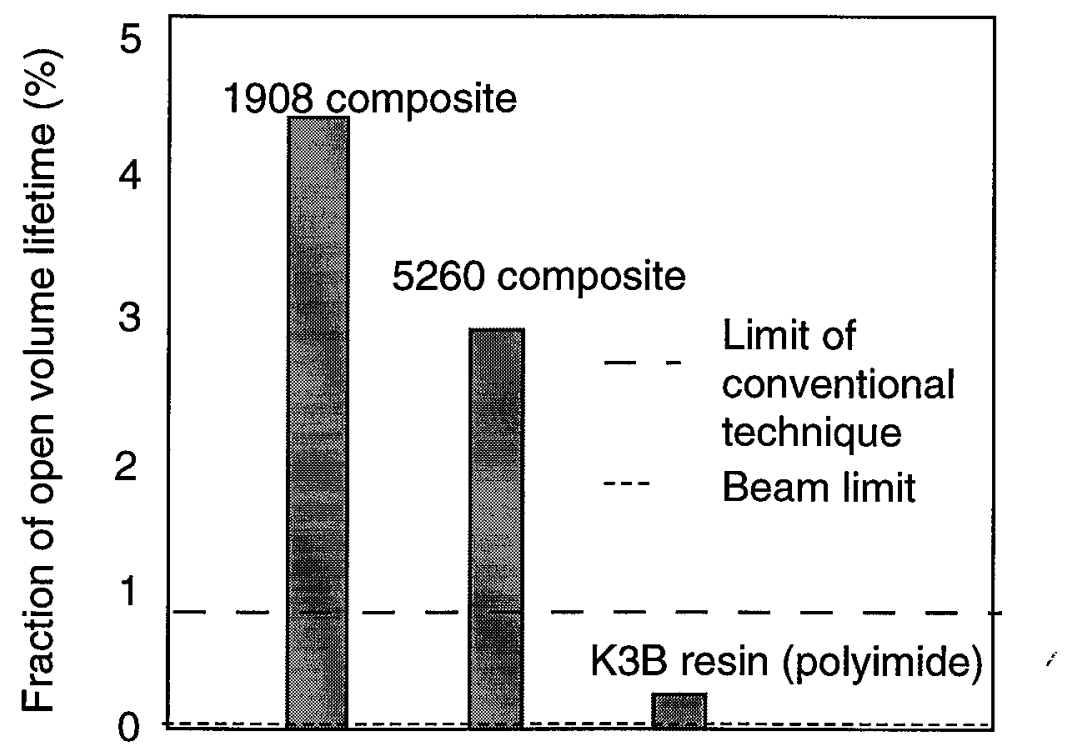

Figure 2. Accurate measurement of positron lifetimes at very low intensities are enabled by eliminating systematic lifetime contributions above $0.25 \mathrm{~ns}$

We are also using the high energy beam lifetime spectrometer to determine in radiation damage in metals from induced radiation from high energy accelerator beams and from self irradiation in radioactive species. Interpretation of lifetimes found in the irradiated alloys is aided by first principles calculations of the bulk and defect lifetimes.

This work was performed under the auspices of the US Department of Energy by LLNL under contract No. W-7405-ENG-48.

1. W. Bauer, J. Briggmann, H.-D. Carstanjen, S. H. Connell, W. Decker, J. Diehl, K. Maier, J. Major, H. E. Schaefer, A. Seeger, H. Stoll and E. Widmann, Nucl. Inst. and Meth. $\underline{\mathrm{B} 50}, 300(1990)$ 

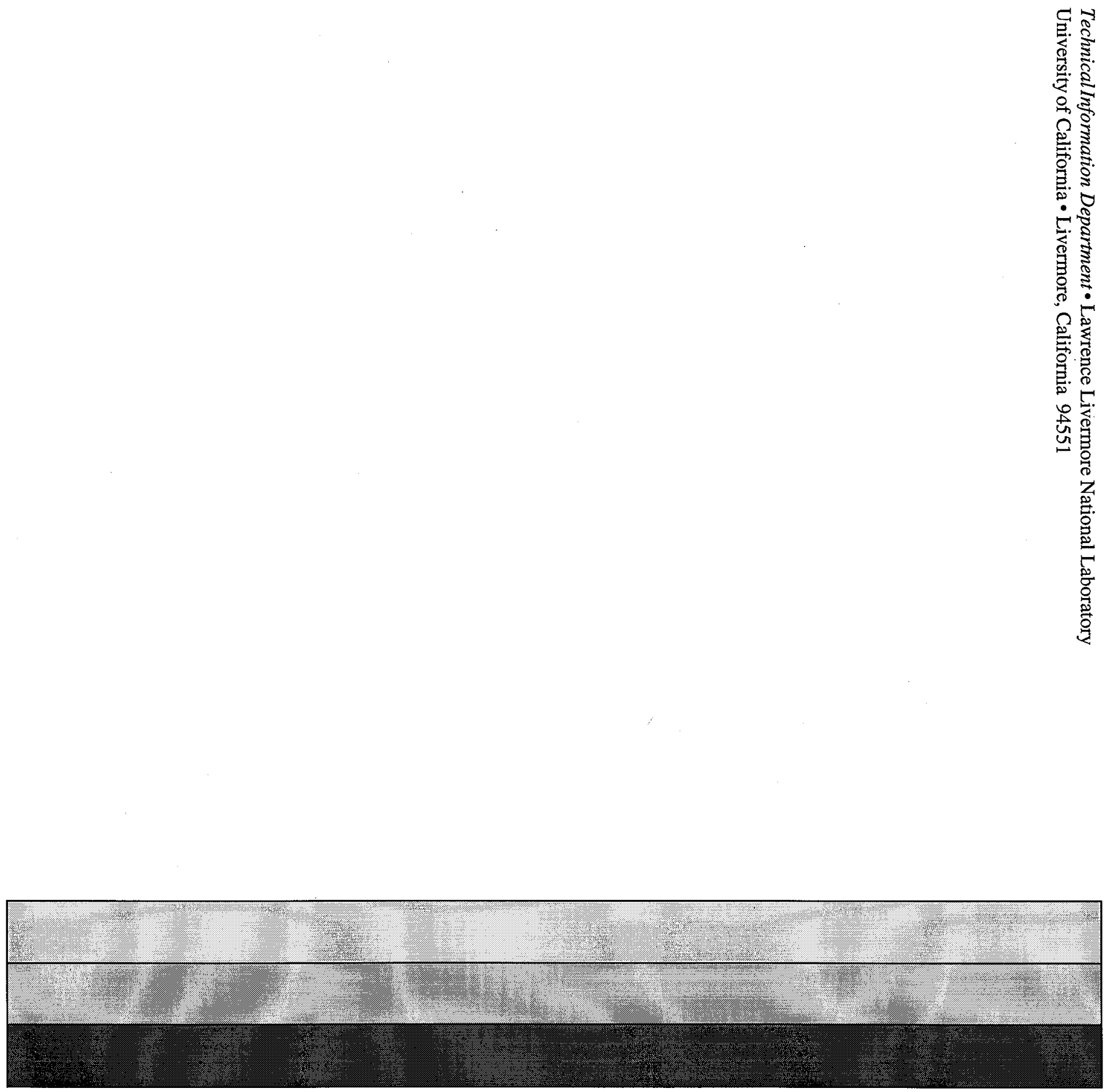